\title{
Rotation algebras and Exel trace formula
}

\author{
Jiajie Hua and Huaxin Lin
}

\begin{abstract}
We found that if $u$ and $v$ are any two unitaries in a unital $C^{*}$-algebra with $\|u v-v u\|<2$ and $u v u^{*} v^{*}$ commutes with $u$ and $v$, then the $C^{*}$-subalgebra $A_{u, v}$ generated by $u$ and $v$ is isomorphic to a quotient of the rotation algebra $A_{\theta}$ provided that $A_{u, v}$ has a unique tracial state. We also found that the Exel trace formula holds in any unital $C^{*}$-algebra. Let $\theta \in(-1 / 2,1 / 2)$ be a real number. We prove the following: For any $\epsilon>0$, there exists $\delta>0$ satisfying the following: if $u$ and $v$ are two unitaries in any unital simple $C^{*}$-algebra $A$ with tracial rank zero such that

$$
\left\|u v-e^{2 \pi i \theta} v u\right\|<\delta \text { and } \frac{1}{2 \pi i} \tau\left(\log \left(u v u^{*} v^{*}\right)\right)=\theta,
$$

for all tracial state $\tau$ of $A$, then there exists a pair of unitaries $\tilde{u}$ and $\tilde{v}$ in $A$ such that

$$
\tilde{u} \tilde{v}=e^{2 \pi i \theta} \tilde{v} \tilde{u},\|u-\tilde{u}\|<\epsilon \text { and }\|v-\tilde{v}\|<\epsilon .
$$
\end{abstract}

\section{Introduction}

Let $\theta$ be a real number in $(-1 / 2,1 / 2)$ and let $A_{\theta}$ be the rotation algebra, which is the universal $C^{*}$-algebra generated by a pair of unitaries $u_{\theta}$ and $v_{\theta}$ subject to the relation that $u_{\theta} v_{\theta}=$ $e^{2 \pi i \theta} v_{\theta} u_{\theta}$. Let $A$ be a unital $C^{*}$-algebra and let $u$ and $v$ be two unitaries with $\|u v-v u\|<2$. Consider the $C^{*}$-subalgebra $A_{u, v}$ generated by $u$ and $v$. A question that one might ask is when $A_{u, v}$ is isomorphic to a quotient of $A_{\theta}$ if $u v u^{*} v^{*}$ commutes with $u$ and $v$. This may seem like a rather unreasonable question, however if $A_{u, v}$ has a unique tracial state, the answer is always "yes", which has a simple proof.

This brings us to the following question:

(Q1): Let $\epsilon>0$. Is there a $\delta>0$ satisfying the following: if $u$ and $v$ are two unitaries in a unital simple $C^{*}$-algebra $A$ with tracial rank zero satisfying

$$
\begin{aligned}
& \left\|u v-e^{2 \pi i \theta} v u\right\|<\delta \text { and } \\
& \frac{1}{2 \pi i} \tau\left(\log \left(u v u^{*} v^{*}\right)\right)=\theta,
\end{aligned}
$$

then there exists a pair of unitaries $\tilde{u}$ and $\tilde{v}$ in $A$ such that

$$
\tilde{u} \tilde{v}=e^{2 \pi i \theta} \tilde{v} \tilde{u}, \quad\|u-\tilde{u}\|<\epsilon \text { and }\|v-\tilde{v}\|<\epsilon ?
$$

Note that $\delta$ is a universal constant independent of $u, v$ and $C^{*}$-algebra $A$.

A related old problem from the 1950s, popularized by Halmos, asks: if a pair of hermitian matrices almost commute, then are they necessarily close to a pair of commuting hermitian matrices [1, 5, 16, 31]? Voiculescu realized that the answer is negative if the word hermitian is replaced by unitary. In fact, Voiculescu showed that, when $\theta=0$, something like (e1.1) in question $(\mathbf{Q} 1)$ is necessary.

Despite Voiculescu's example, however, the related problem about almost commuting hermitian was solved affirmatively by the second named author in [20] (see also [14 for a simplified 
exposition). The problem whether a pair of almost commuting unitaries can be approximated by a pair of commuting unitaries had been further studied by [3, 5, 27, 12, 13, and others. Exel and Loring ([13]) showed that the condition (e1.1) is necessary for $(\mathbf{Q} 1)$ in the case that $\theta=0$ following Voiculescu's example and they recognized that the obstacle in Voiculescu's example is the bott element. Things moved fast in the middle of 1990's with the proof in ([20]). It has been proved that $(\mathbf{Q} 1)$ has an affirmative answer when $\theta=0$ (see [15], 28] and [8]). The trace formula for the bott element provided by Exel ([11]) is a very convenient tool. In fact, the recent development in the connection to the Elliott program of classification of amenable $C^{*}$-algebras shows that the Exel trace formula has many further applications. The Exel trace formula brought the bott element, a topological obstruction, and rotation number, a dynamical description together. Originally, the Exel trace formula was proved in matrix algebras ([11]). We note that it in fact holds in general $C^{*}$-algebras. One might say that this paper is a further understanding of the Exel trace formula in the context of rotation algebras.

Shortly after the first version of these notes posted, Terry Loring informed us his joint work on (Q1). In fact, Eilers and Loring showed in [6] that, for rational values in $(-1 / 2,1 / 2)$ (Eilers, Loring and Pedersen showed in [7] that, for rational value $\left.\frac{1}{2}\right)$, the answer to (Q1) is affirmative if the class of all unital simple $C^{*}$-algebras with real rank zero is replaced by class of finite dimensional simple $C^{*}$-algebras. It should be noted that, when $A$ is a matrix algebra $M_{n}$, $\frac{1}{2 \pi i} \tau\left(\log \left(u v u^{*} v^{*}\right)\right)$ is always a rational number. Moreover, when $\theta$ is an irrational number, $A_{\theta}$ is always infinite dimensional. Therefore there is no homomorphisms from $A_{\theta}$ into $M_{n}$. It seems natural to think that the next class of $C^{*}$-algebras to finite dimensional simple $C^{*}$-algebras is the class of unital simple AF-algebras, or more generally, unital simple $C^{*}$-algebras of tracial rank zero (see 4.1 below). We show that the answer to $(\mathbf{Q} 1)$ is in the affirmative.

This paper is organized as follows. In section 2, we list some notations and some known results about certain universal $C^{*}$-algebras generated by two unitaries. In section 3 , we give a proof that the Exel trace formula holds for any unital $C^{*}$-algebra. In section 4 , we show that the answer to question $(\mathbf{Q} 1)$ is in the affirmative for irrational numbers $\theta$. In the last section, we show that, when $\theta$ is rational, we also have an affirmative answer to a version of $(\mathbf{Q} 1)$. In fact, we allow a somewhat larger class of $C^{*}$-algebras, i.e., the class of unital simple $C^{*}$-algebras of real rank zero and stable rank one.

\section{Preliminaries}

All statements in this section are known. We list here for the convenience.

Definition 2.1. Let $A$ be a unital $C^{*}$-algebra. Denote by $T(A)$ the tracial state space of $A$. We will use $\tau$ for $\tau \otimes \operatorname{Tr}$ on $M_{n}(A)$, where $\operatorname{Tr}$ is the standard trace on $M_{n}, n=1,2, \ldots$ Denote by $\rho_{A}: K_{0}(A) \rightarrow \operatorname{Aff}(T(A))$ the order preserving map induced by $\rho_{A}([p])(\tau)=\tau(p)$ for all projections $p \in A \otimes M_{n}, n=1,2, \ldots$

Definition 2.2. Let $A$ be a unital $C^{*}$-algebra and let $u \in A$ be a unitary. Define $\operatorname{Ad} u(a)=u^{*} a u$ for all $a \in A$.

The $C^{*}$-algebra $C\left(\mathbb{T}^{2}\right)$ of all continuous complex valued functions on the two-torus is well known to be the universal $C^{*}$-algebra generated by two commuting unitary elements.

Definition 2.3. Let $\epsilon \in[0,2)$. Recall that the "Soft Torus" $\mathcal{T}_{\epsilon}$ is the universal $C^{*}$-algebra generated by a pair of elements $\mathfrak{u}_{\epsilon}$ and $\mathfrak{v}_{\epsilon}$, subject to the relations $\mathfrak{u}_{\epsilon}^{*} \mathfrak{u}_{\epsilon}=\mathfrak{u}_{\epsilon} \mathfrak{u}_{\epsilon}^{*}=1=\mathfrak{v}_{\epsilon}^{*} \mathfrak{v}_{\epsilon}=\mathfrak{v}_{\epsilon} \mathfrak{v}_{\epsilon}^{*}$ and $\left\|\mathfrak{u}_{\epsilon} \mathfrak{v}_{\epsilon}-\mathfrak{v}_{\epsilon} \mathfrak{u}_{\epsilon}\right\| \leq \epsilon$.

Given $\theta \in \mathbb{R}$, let $A_{\theta}$ be the universal $C^{*}$-algebra generated by a pair of elements $u_{\theta}$ and $v_{\theta}$, subject to the relations $u_{\theta}^{*} u_{\theta}=u_{\theta} u_{\theta}^{*}=1=v_{\theta}^{*} v_{\theta}=v_{\theta} v_{\theta}^{*}$ and $u_{\theta} v_{\theta}=e^{2 \pi i \theta} v_{\theta} u_{\theta}$. If $\theta$ is irrational 
(rational), $A_{\theta}$ is called the irrational (rational) rotation algebra. The algebras $A_{\theta}$ are usually called noncommutative tori, since $C\left(\mathbb{T}^{2}\right) \cong A_{0}$.

Let $B_{\epsilon}$ be the universal $C^{*}$-algebra generated by a countable set $\left\{x_{n}: n \in \mathbb{Z}\right\}$ subject to the conditions that each $x_{n}$ is unitary and that $\left\|x_{n+1}-x_{n}\right\| \leq \epsilon$ for all $n$.

Let $\alpha_{\epsilon}$ be the automorphism of $B_{\epsilon}$ specified by $\alpha_{\epsilon}\left(x_{n}\right)=x_{n+1}$. More details for the "Soft Torus" $\mathcal{T}_{\epsilon}$ and $B_{\epsilon}$ can be found in [11].

Theorem 2.4 (Theorem 2.2 of [11]). Let $z$ denote the canonical generator of the algebra $C(\mathbb{T})$ of continuous functions on the unit circle, and let $\psi_{\epsilon}: B_{\epsilon} \rightarrow C(\mathbb{T})$ be the unique homomorphism such that $\psi_{\epsilon}\left(x_{n}\right)=z$ for all $n$. If $\epsilon<2$, then $\psi_{\epsilon}$ is a homotopy equivalence between $B_{\epsilon}$ and $C(\mathbb{T})$.

Proposition 2.5 (Proposition 2.3 of [11]). For all $\epsilon \in[0,2)$ one has an isomorphism $\varphi: \mathcal{T}_{\epsilon} \rightarrow$ $B_{\epsilon} \rtimes_{\alpha_{\epsilon}} \mathbb{Z}$ such that $\varphi\left(\mathfrak{u}_{\epsilon}\right)=x_{0}$ and $\varphi\left(\mathfrak{v}_{\epsilon} \mathfrak{u}_{\epsilon} \mathfrak{v}_{\epsilon}^{*}\right)=x_{1}$.

This is proved in Proposition 2.3 of [11]. We would like to bring the attention that $\varphi\left(\mathfrak{u}_{\epsilon}\right)=x_{0}$ and $\varphi\left(\mathfrak{v}_{\epsilon} \mathfrak{u}_{\epsilon} \mathfrak{v}_{\epsilon}^{*}\right)=x_{1}$.

In what follows we will identity $x_{0}$ with $\mathfrak{u}_{\epsilon}$ and $x_{1}=\mathfrak{v}_{\epsilon} \mathfrak{u}_{\epsilon} \mathfrak{v}_{\epsilon}^{*}$.

Let $z$ and $w$ denote the coordinate functions on the two-torus so that $z$ and $w$ represent two unitaries in $C\left(\mathbb{T}^{2}\right)$. There is a unital homomorphism $\varphi_{\epsilon}: \mathcal{T}_{\epsilon} \rightarrow C\left(\mathbb{T}^{2}\right)$ such that $\varphi_{\epsilon}\left(\mathfrak{u}_{\epsilon}\right)=z$ and $\varphi_{\epsilon}\left(\mathfrak{v}_{\epsilon}\right)=w$.

By the proof of Theorem 2.4 of [11], we have the following commutative diagram with exact rows.

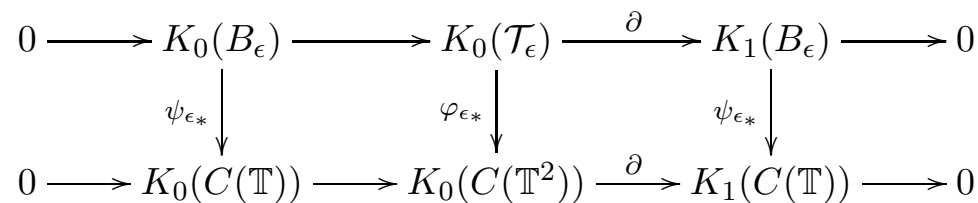

where $\psi_{\epsilon_{*}}$ and $\varphi_{\epsilon_{*}}$ are isomorphisms.

Definition 2.6. By the above diagram, there is an element $b \in K_{0}\left(C\left(\mathbb{T}^{2}\right)\right)$ such that $\partial(b)=[z]$ in $K_{1}(C(\mathbb{T}))$. Denote by $b_{\epsilon}$ the element in $K_{0}\left(\mathcal{T}_{\epsilon}\right)$ defined by $b_{\epsilon}=\varphi_{\epsilon_{*}}^{-1}(b)$, then $\partial\left(b_{\epsilon}\right)=\left[x_{0}\right]=\left[\mathfrak{u}_{\epsilon}\right]$ in $K_{1}\left(B_{\epsilon}\right)$.

We may assume that there are projections $p_{\epsilon}, q_{\epsilon} \in M_{K}\left(\mathcal{T}_{\epsilon}\right)$ such that $\left[p_{\epsilon}\right]-\left[q_{\epsilon}\right]=b_{\epsilon}$, where $K$ is an integer. Note that

$$
\left|\tau \circ \rho_{\mathcal{T}_{\epsilon}}\left(b_{\epsilon}\right)\right| \leq 2 K
$$

for all $\tau \in T\left(\mathcal{T}_{\epsilon}\right)$.

Definition 2.7. Define

$$
\begin{gathered}
f\left(e^{2 \pi i t}\right)= \begin{cases}1-2 t, & \text { if } 0 \leq t \leq 1 / 2, \\
-1+2 t, & \text { if } 1 / 2<t \leq 1,\end{cases} \\
g\left(e^{2 \pi i t}\right)= \begin{cases}\left(f\left(e^{2 \pi i t}\right)-f\left(e^{2 \pi i t}\right)^{2}\right)^{\frac{1}{2}}, & \text { if } 0 \leq t \leq 1 / 2, \\
0, & \text { if } 1 / 2<t \leq 1,\end{cases} \\
h\left(e^{2 \pi i t}\right)= \begin{cases}0, & \text { if } 0 \leq t \leq 1 / 2, \\
\left(f\left(e^{2 \pi i t}\right)-f\left(e^{2 \pi i t}\right)^{2}\right)^{\frac{1}{2}}, & \text { if } 1 / 2<t \leq 1 .\end{cases}
\end{gathered}
$$

These are non-negative continuous functions defined on the unit circle.

Let $A$ be a unital $C^{*}$-algebra and $u, v \in A$ be two unitaries, define

$$
e(u, v)=\left(\begin{array}{cc}
f(u) & g(u)+h(u) v^{*} \\
g(u)+v h(u) & 1-f(u)
\end{array}\right) .
$$


It is a positive element. Suppose that $u v=v u$, then $e(u, v)$ is a projection.

In $M_{2}\left(C\left(\mathbb{T}^{2}\right)\right), e(z, w)$ is a non-trivial rank one projection. Then

$$
b=[e(z, w)]-\left[\left(\begin{array}{ll}
1 & 0 \\
0 & 0
\end{array}\right)\right]
$$

is often called the bott element for $C\left(\mathbb{T}^{2}\right)$.

There is $\delta_{0}>0$ (independent of unitaries $u, v$ and $A$ ) such that if $\|[u, v]\|<\delta_{0}$, then the spectrum of the positive element $e(u, v)$ has a gap at $1 / 2$. The bott element of $u$ and $v$ is an element in $K_{0}(A)$ as defined by

$$
\operatorname{bott}(u, v)=\left[\chi_{1 / 2, \infty}(e(u, v))\right]-\left[\left(\begin{array}{ll}
1 & 0 \\
0 & 0
\end{array}\right)\right] .
$$

Note that (when $\|u v-v u\|<\delta_{0}$ ) there is a continuous function $\chi:[0, \infty) \rightarrow[0,1]$ such that

$$
\chi(e(u, v))=\chi_{(1 / 2, \infty)}(e(u, v)) .
$$

The reader is referred to [12], [13] and [27] for more information about the bott element.

The following is also known.

Proposition 2.8. If $\epsilon \in\left[0, \delta_{0}\right)$, then

$$
b_{\epsilon}=\operatorname{bott}\left(\mathfrak{u}_{\epsilon}, \mathfrak{v}_{\epsilon}\right) .
$$

Proof. When $\epsilon \in\left[0, \delta_{0}\right)$,

$$
\left(\varphi_{\epsilon} \otimes \operatorname{id}_{M_{2}}\right)\left(\chi\left(e\left(\mathfrak{u}_{\epsilon}, \mathfrak{v}_{\epsilon}\right)\right)\right)=\chi\left(e\left(\varphi_{\epsilon}\left(\mathfrak{u}_{\epsilon}\right), \varphi_{\epsilon}\left(\mathfrak{v}_{\epsilon}\right)\right)\right)=\chi(e(z, w))=e(z, w) .
$$

It follows that

$$
\varphi_{\epsilon_{*}}\left(\left[\chi\left(e\left(\mathfrak{u}_{\epsilon}, \mathfrak{v}_{\epsilon}\right)\right)\right]-\left[\left(\begin{array}{cc}
1 & 0 \\
0 & 0
\end{array}\right)\right]\right)=b
$$

Therefore

$$
b_{\epsilon}=\operatorname{bott}\left(\mathfrak{u}_{\epsilon}, \mathfrak{v}_{\epsilon}\right)
$$

\section{Exel trace formula}

Let $A$ be a unital $C^{*}$-algebra and let $\alpha: A \rightarrow A$ be an automorphism. If $\tau$ is a trace on $A$ which is invariant under the action $\alpha$ and if $u$ is implementing unitary of $\alpha$, then $\tau \circ E$ gives a trace $\tilde{\tau}$ on $A \rtimes_{\alpha} \mathbb{Z}$, where $E: A \rtimes_{\alpha} \mathbb{Z} \rightarrow A$ is the expectation defined by $E\left(\sum_{i=-n}^{n} a_{i} u^{i}\right)=a_{0}$.

Definition 3.1 (Definition II.9 of [10]). If $\tau \in T(A)$ is a fixed tracial state on a unital $C^{*}$-algebra $A$, we say that the pair $(A, \tau)$ is an integral $C^{*}$-algebra if $\rho_{A}(x)(\tau) \subset \mathbb{Z}$ for all $x \in K_{0}(A)$.

Let $A$ be a unital $C^{*}$-algebra and $U_{n}(A)$ be the group of all unitary elements of $A \otimes M_{n}$, $n=1,2, \ldots$. We denote by $U_{\infty}(A)$ the inductive limit of the sequence of groups

$$
U_{1}(A) \stackrel{i_{1}}{\rightarrow} U_{2}(A) \stackrel{i_{2}}{\rightarrow} \cdots U_{n}(A) \stackrel{i_{n}}{\rightarrow} U_{n+1}(A) \stackrel{i_{n+1}}{\rightarrow} \cdots
$$

where $i_{n}$ is defined by

$$
i_{n}(u)=u \oplus 1_{A} \in U_{n+1}(A) \text { for all } u \in U_{n}(A) \text { and all } n \in \mathbb{N} .
$$

We often use $U(A)$ for $U_{1}(A)$. 
Definition 3.2 (Definition II.2 of [10] and also see [17]). Let $A$ be a unital $C^{*}$-algebra and let $\tau \in T(A)$. We say that a group homomorphism

$$
\operatorname{det}_{\tau}: U_{\infty}(A) \rightarrow \mathbb{T}
$$

is a determinant associated with the tracial state $\tau$ if for all self-adjoint elements $h \in M_{n}(A)$, one has

$$
\operatorname{det}_{\tau}\left(e^{i h}\right)=e^{i \tau(h)} .
$$

It is proved by Exel (Theorem II.10 of [10]) that such determinant exists if and only if $(A, \tau)$ is an integral $C^{*}$-algebra.

Let $\alpha$ be an automorphism of a unital $C^{*}$-algebra $A$. Denote by $\partial: K_{0}\left(A \rtimes_{\alpha} \mathbb{Z}\right) \rightarrow K_{1}(A)$ the connecting map of the Pimsner-Voiculescu sequence ([29]).

Let us recall the following two results.

Theorem 3.3 (Theorem V.12 of [10]). Let $(A, \tau)$ be an integral unital $C^{*}$-algebra and $\alpha$ be a trace-preserving automorphism of $A$. Then for every a in $K_{0}\left(A \rtimes_{\alpha} \mathbb{Z}\right)$, we have

$$
\exp \left(2 \pi i \tilde{\tau} \circ \rho_{A \rtimes_{\alpha} \mathbb{Z}}(a)\right)=\operatorname{det}_{\tau}\left(\alpha\left(u^{-1}\right) u\right),
$$

where $u$ is any unitary element of $U_{\infty}(A)$ whose $K_{1}$-class is $\partial(a)$.

Lemma 3.4 (Lemma 3.3 of [11]). Let $\alpha$ be an automorphism of a $C^{*}$-algebra $A$ and let $\tau_{1}$ and $\tau_{2}$ be traces on $A \rtimes_{\alpha} \mathbb{Z}$ such that $\tau_{1}=\tau_{2}$ on $A$. Then $\tau_{1} \circ \rho_{A \rtimes_{\alpha} \mathbb{Z}}=\tau_{2} \circ \rho_{A \rtimes_{\alpha} \mathbb{Z}}$ on $K_{0}\left(A \rtimes_{\alpha} \mathbb{Z}\right)$.

Note that if $\epsilon \in[0,2),\left\|u v u^{*} v^{*}-1\right\|=\epsilon<2$. Then -1 is not in the spectrum of $u v u^{*} v^{*}$. Therefore there is a continuous branch of logarithm defined on the compact subset $F_{\epsilon}=\left\{e^{i t}\right.$ : $t \in[-\pi+2 \arccos (\epsilon / 2), \pi-2 \arccos (\epsilon / 2)]\}$. In what follows, unless otherwise state, we use $\log$ defined on $F_{\epsilon}$. Moreover, if $0<\epsilon_{1}<\epsilon$, we may assume that $\log$ is defined on $F_{\epsilon}$.

Theorem 3.5. Let $\epsilon \in[0,2), \mathfrak{u}_{\epsilon}, \mathfrak{v}_{\epsilon} \in U\left(\mathcal{T}_{\epsilon}\right)$ be generators of $\mathcal{T}_{\epsilon}$, then

$$
\rho_{\mathcal{T}_{\epsilon}}\left(b_{\epsilon}\right)(\tau)=\frac{1}{2 \pi i} \tau\left(\log \left(\mathfrak{u}_{\epsilon} \mathfrak{v}_{\epsilon} \mathfrak{u}_{\epsilon}^{*} \mathfrak{v}_{\epsilon}^{*}\right)\right) \quad \text { for all } \quad \tau \in T\left(\mathcal{T}_{\epsilon}\right) .
$$

In particular, when $\epsilon \in\left[0, \delta_{0}\right)$,

$$
\rho_{\mathcal{T}_{\epsilon}}\left(\operatorname{bott}\left(\mathfrak{u}_{\epsilon}, \mathfrak{v}_{\epsilon}\right)\right)(\tau)=\frac{1}{2 \pi i} \tau\left(\log \left(\mathfrak{u}_{\epsilon} \mathfrak{v}_{\epsilon} \mathfrak{u}_{\epsilon}^{*} \mathfrak{v}_{\epsilon}^{*}\right)\right) \quad \text { for all } \quad \tau \in T\left(\mathcal{T}_{\epsilon}\right) .
$$

Proof. Identify $B_{\epsilon}$ as a subalgebra of $\mathcal{T}_{\epsilon}$ under the isomorphism of Proposition 2.5. Let $\tau \in T\left(\mathcal{T}_{\epsilon}\right)$. Then $\tau$ is given by restriction an $\alpha_{\epsilon}$-invariant trace on $B_{\epsilon}$. Moreover, $\tau$ is an integral trace on $B_{\epsilon}$ since any tracial state is an integral trace on the homotopy class of $C(\mathbb{T})$ by Theorem 2.4 . Let $\tilde{\tau}$ be the canonical extension of $\left.\tau\right|_{B_{\epsilon}}$, by Theorem 3.4 and Theorem 3.3 we obtain

$$
\begin{aligned}
\exp \left(2 \pi i \tau \circ \rho_{\mathcal{T}_{\epsilon}}\left(b_{\epsilon}\right)\right) & =\exp \left(2 \pi i \tilde{\tau} \circ \rho_{\mathcal{T}_{\epsilon}}\left(b_{\epsilon}\right)\right) \\
& =\operatorname{det}_{\tau}\left(\alpha_{\epsilon}\left(\mathfrak{u}_{\epsilon}^{*}\right) \mathfrak{u}_{\epsilon}\right) \\
& =\operatorname{det}_{\tau}\left(\alpha_{\epsilon}\left(x_{0}^{*}\right) x_{0}\right) \\
& =\operatorname{det}_{\tau}\left(x_{1}^{*} x_{0}\right) \\
& =\operatorname{det}_{\tau}\left(\exp \left(\log \left(x_{1}^{*} x_{0}\right)\right)\right) \\
& =\exp \left(\tau\left(\log \left(x_{1}^{*} x_{0}\right)\right)\right) \\
& =\exp \left(\tau\left(\log \left(\mathfrak{v}_{\epsilon} \mathfrak{u}_{\epsilon}^{*} \mathfrak{v}_{\epsilon}^{*} \mathfrak{u}_{\epsilon}\right)\right)\right) \\
& =\exp \left(\tau\left(\log \left(\mathfrak{u}_{\epsilon} \mathfrak{v}_{\epsilon} \mathfrak{u}_{\epsilon}^{*} \mathfrak{v}_{\epsilon}^{*}\right)\right)\right) .
\end{aligned}
$$


So there is an integer $k_{\tau} \in \mathbb{Z}$ such that

$$
\rho_{\mathcal{T}_{\epsilon}}\left(b_{\epsilon}\right)(\tau)-\frac{1}{2 \pi i} \tau\left(\log \left(\mathfrak{u}_{\epsilon} \mathfrak{v}_{\epsilon} \mathfrak{u}_{\epsilon}^{*} \mathfrak{v}_{\epsilon}^{*}\right)\right)=k_{\tau} .
$$

Note by (e2.2),

$$
\left|k_{\tau}\right| \leq 2 K+1 \text { for all } \tau \in T\left(\mathcal{T}_{\epsilon}\right) .
$$

Let $u=\mathfrak{u}_{\epsilon} \oplus I_{m}$ and $v=\mathfrak{v}_{\epsilon} \oplus I_{m}$, where $I_{m}$ is the identity of $M_{m}\left(\mathcal{T}_{\epsilon}\right)$, then $\|u v-v u\| \leq \epsilon$. Since $\mathcal{T}_{\epsilon}$ is universal, we have a homomorphism $\Phi: \mathcal{T}_{\epsilon} \rightarrow M_{m+1}\left(\mathcal{T}_{\epsilon}\right)$ such that $\Phi\left(\mathfrak{u}_{\epsilon}\right)=u$ and $\Phi\left(\mathfrak{v}_{\epsilon}\right)=v$.

Fix $\xi=(1,1) \in \mathbb{T} \times \mathbb{T}=\mathbb{T}^{2}$. Let $P_{\xi}: C\left(\mathbb{T}^{2}\right) \rightarrow \mathbb{C}$ be the point-evaluation defined by $P_{\xi}(f)=f(\xi)$ for all $f \in C\left(\mathbb{T}^{2}\right)$. Denote by $\pi_{\xi}: \mathcal{T}_{\epsilon} \rightarrow \mathbb{C}$ by $\pi_{\xi}=P_{\xi} \circ \varphi_{\epsilon}$, where $\varphi_{\epsilon}: \mathcal{T}_{\epsilon} \rightarrow C\left(\mathbb{T}^{2}\right)$ is defined right before 2.6. Note $\left(\pi_{\xi}\right)_{* 0}\left(b_{\epsilon}\right)=0$. Note

$$
\Phi(a)=\left(\begin{array}{cccc}
a & 0 & 0 & \ldots \\
0 & \pi_{\xi}(a) & 0 & \ldots \\
& & \ddots & \\
& & & \pi_{\xi}(a)
\end{array}\right)
$$

for all $a \in \mathcal{T}_{\epsilon}$.

Let $\tau_{0} \in T\left(M_{m+1}\left(\mathcal{T}_{\epsilon}\right)\right)$. Then $\tau_{0} \circ \Phi$ is an an integral trace on $B_{\epsilon}$ since any tracial state is an integral trace on the homotopy class of $C(\mathbb{T})$ by Theorem 2.4. It follows that

$$
\tau_{0} \circ \rho_{M_{m+1}\left(\mathcal{T}_{\epsilon}\right)}\left(\Phi_{* 0}\left(b_{\epsilon}\right)\right)-\frac{1}{2 \pi i} \tau_{0} \circ \Phi\left(\log \left(\mathfrak{u}_{\epsilon} \mathfrak{v}_{\epsilon} \mathfrak{u}_{\epsilon}^{*} \mathfrak{v}_{\epsilon}^{*}\right)\right)=k_{\tau_{0} \circ \Phi} \in \mathbb{Z} .
$$

On the other hand, one may write $\tau_{0}=\frac{1}{m+1}(\tau \oplus \cdots \oplus \tau)$ for some tracial state $\tau \in T\left(\mathcal{T}_{\epsilon}\right)$. We compute that

$$
\tau_{0} \circ \Phi\left(\mathfrak{u}_{\epsilon} \mathfrak{v}_{\epsilon} \mathfrak{u}_{\epsilon}^{*} \mathfrak{v}_{\epsilon}^{*}\right)=\frac{1}{m+1} \tau\left(\log \left(\mathfrak{u}_{\epsilon} \mathfrak{v}_{\epsilon} \mathfrak{u}_{\epsilon}^{*} \mathfrak{v}_{\epsilon}^{*}\right)\right)
$$

By the definition of $\Phi$ and the fact that $\left(\pi_{\xi}\right)_{* 0}\left(b_{\epsilon}\right)=0$, we also have

$$
\tau_{0} \circ \rho_{M_{m+1}\left(\mathcal{T}_{\epsilon}\right)}\left(\Phi_{* 0}\left(b_{\epsilon}\right)\right)=\frac{1}{m+1} \tau \circ \rho_{\mathcal{T}_{\epsilon}}\left(b_{\epsilon}\right) .
$$

It follows, by combinning (e 2.2) that

$$
\left|k_{\tau_{0} \circ \Phi}\right|=\left|\frac{k_{\tau}}{m+1}\right| \leq \frac{2 K+1}{m+1} .
$$

This holds for all integer $m$. It follows that $k_{\tau_{0} \circ \Phi}=0$. Then, by (e3.3), $k_{\tau}=0$ for all $\tau \in T\left(\mathcal{T}_{\epsilon}\right)$. Therefore

$$
\rho_{\mathcal{T}_{\epsilon}}\left(b_{\epsilon}\right)(\tau)=\frac{1}{2 \pi i} \tau\left(\log \left(\mathfrak{u}_{\epsilon} \mathfrak{v}_{\epsilon} \mathfrak{u}_{\epsilon}^{*} \mathfrak{v}_{\epsilon}^{*}\right)\right) \quad \text { for all } \quad \tau \in T\left(\mathcal{T}_{\epsilon}\right)
$$

Definition 3.6. Let $A$ be a unital $C^{*}$-algebra and let $u$ and $v$ be two unitaries in $A$ such that $\|u v-v u\| \leq \epsilon<2$. Denote by $A_{u, v}$ the $C^{*}$-subalgebra of $A$ generated by $u$ and $v$. There is a surjective homomorphism $\phi_{u, v}: \mathcal{T}_{\epsilon} \rightarrow A_{u, v}$ such that $\phi_{u, v}\left(\mathfrak{u}_{\epsilon}\right)=u$ and $\phi_{u, v}\left(\mathfrak{v}_{\epsilon}\right)=v$. Put $b_{u, v}=\left(\phi_{u, v}\right)_{* 0}\left(b_{\epsilon}\right)$. If $\|u v-v u\|<\delta_{0}$, then

$$
b_{u, v}=\operatorname{bott}(u, v) .
$$


Theorem 3.7. (Exel trace formula) Let $A$ be a unital $C^{*}$-algebra, then for any $u, v \in U(A)$ and $\|u v-v u\|<2$, we have

$$
\rho_{A}\left(\imath_{* 0}\left(b_{u, v}\right)\right)(\tau)=\frac{1}{2 \pi i} \tau\left(\log \left(u v u^{*} v^{*}\right)\right) \quad \text { for all } \quad \tau \in T(A),
$$

where $\imath: A_{u, v} \rightarrow A$ is the unital embedding. If, in addition, $\|u v-v u\|<\delta_{0}$, then

$$
\rho_{A}(\operatorname{bott}(u, v))(\tau)=\frac{1}{2 \pi i} \tau\left(\log \left(u v u^{*} v^{*}\right)\right) \quad \text { for all } \quad \tau \in T(A)
$$

Proof. Since $\|u v-v u\|=\epsilon<2$, there is a unique homomorphism $\phi: \mathcal{T}_{\epsilon} \rightarrow A$ such that $\phi\left(\mathfrak{u}_{\epsilon}\right)=u$ and $\phi\left(\mathfrak{v}_{\epsilon}\right)=v$, then $\tau \circ \phi$ is a tracial state on $\mathcal{T}_{\epsilon}$, we get

$$
\rho_{\mathcal{T}_{\epsilon}}\left(b_{\epsilon}\right)(\tau \circ \phi)=\frac{1}{2 \pi i} \tau \circ \phi\left(\log \left(\mathfrak{u}_{\epsilon} \mathfrak{v}_{\epsilon} \mathfrak{u}_{\epsilon}^{*} \mathfrak{v}_{\epsilon}^{*}\right)\right) \quad \text { for all } \quad \tau \in T(A) .
$$

Note that $\phi\left(\mathcal{T}_{\epsilon}\right)=A_{u, v}$. So

$$
\rho_{A}\left(l_{* 0}\left(b_{u, v}\right)\right)(\tau)=\frac{1}{2 \pi i} \tau\left(\log \left(u v u^{*} v^{*}\right)\right) \quad \text { for all } \quad \tau \in T(A) .
$$

Remark 3.8. The Exel trace formula was first found for matrix algebras ([11]). It was late proved that the same formula also holds in unital simple $C^{*}$-algebras of tracial rank no more than one (Theorem 3.5 of [26]).

Theorem 3.9. Let $A$ be a unital $C^{*}$-algebra and $u, v \in U(A)$ satisfy the condition $\|u v-v u\|=$ $\delta<2$. Let $A_{u, v}$ be the $C^{*}$-subalgebra generated by unitaries $u$ and $v$ such that uvu* $v^{*}$ commutes with $u$ and $v$. Suppose that $\theta \in(-1 / 2,1 / 2)$. If $T\left(A_{u, v}\right) \neq \emptyset$ and $\frac{1}{2 \pi i} \tau\left(\log \left(u v u^{*} v^{*}\right)\right)=\theta$ for all $\tau \in T\left(A_{u, v}\right)$, then $A_{u, v}$ is isomorphic to a quotient of $A_{\theta}$. Moreover, uv $=e^{2 \pi i \theta} v u$.

In particular, if $\theta$ is an irrational number, then $A_{u, v} \cong A_{\theta}$.

Proof. Let $\epsilon=\max \left\{\delta,\left|1-e^{2 \pi i \theta}\right|\right\}$. Then $2>\epsilon>0$. Since $\mathcal{T}_{\epsilon}$ is a universal $C^{*}$-algebra, there is a unital surjective homomorphisms $\phi_{u, v}: \mathcal{T}_{\epsilon} \rightarrow A_{u, v}$ such that $\phi_{u, v}\left(\mathfrak{u}_{\epsilon}\right)=u$ and $\phi_{u, v}\left(\mathfrak{v}_{\epsilon}\right)=$ $v$. Moreover, for any $\theta^{\prime} \in(-1 / 2,1 / 2)$ satisfying $\left|1-e^{2 \pi i \theta^{\prime}}\right| \leq \epsilon$, there is a unital surjective homomorphism $\phi_{\theta^{\prime}}: \mathcal{T}_{\epsilon} \rightarrow A_{\theta^{\prime}}$ such that $\phi_{\theta^{\prime}}\left(\mathfrak{u}_{\epsilon}\right)=u_{\theta^{\prime}}$ and $\phi_{\theta^{\prime}}\left(\mathfrak{v}_{\epsilon}\right)=v_{\theta^{\prime}}$.

Let $w_{\epsilon}=\mathfrak{u}_{\epsilon} \mathfrak{v}_{\epsilon} \mathfrak{u}_{\epsilon}^{*} \mathfrak{v}_{\epsilon}^{*}$ and $w=u v u^{*} v^{*}$, so $\phi_{u, v}\left(w_{\epsilon}\right)=w$.

Suppose that the spectrum of $w$ has more than one point, say $e^{2 \pi i \theta_{1}}$ and $e^{2 \pi i \theta_{2}}$, since $\|w-1\|=$ $\left\|u v u^{*} v^{*}-1\right\|=\|u v-v u\| \leq \epsilon$, we have $\left|1-e^{2 \pi i \theta_{j}}\right| \leq \epsilon$ for $j=1,2$.

Note that $w$ commutes with $u$ and $v$. Working in the enveloping von Neumann algebra $A_{u, v}^{* *}$, let $p_{\theta_{j}} \in A_{u, v}^{* *}$ be the spectrum projection of $w$ associated with the point $\left\{e^{2 \pi i \theta_{j}}\right\}, j=1,2$. Since $e^{2 \pi i \theta_{j}}$ is in the spectrum of $w, p_{\theta_{j}} \neq 0$ in $A_{u, v}^{* *}, j=1,2$. Moreover, $p_{\theta_{j}}$ is a closed projection of $A_{u, v}$. Since $w$ commutes with $u$ and $v, p_{\theta_{j}}$ is central. Define $\varphi_{j}(a)=a p_{\theta_{j}}$ for all $a \in A_{u, v}$, $j=1,2$. Then $\varphi_{j}(w)=p_{\theta_{j}} w=e^{2 \pi i \theta_{j}} p_{\theta_{j}}, j=1,2$. It follows that

$$
\varphi_{j}(u) \pi_{j}(v)=p_{\theta_{j}} u v=p_{\theta_{j}} w v u=e^{2 \pi i \theta_{j}} \varphi_{j}(v) \varphi_{j}(u), \quad j=1,2 .
$$

Thus $\varphi_{j}: A_{u, v} \rightarrow \varphi_{j}(A)$ is a unital surjective homomorphism from $A_{u, v}$ onto a quotient of $A_{\theta_{j}}$, $j=1,2$.

Let $\tau_{j} \in T\left(A_{\theta_{j}}\right)$, then $\tau_{j} \circ \varphi_{j} \in T\left(A_{u, v}\right)$. We have

$$
\begin{aligned}
& \frac{1}{2 \pi i}\left(\tau_{j} \circ \varphi_{j}\left(\log \left(u v u^{*} v^{*}\right)\right)\right) \\
= & \frac{1}{2 \pi i}\left(\tau_{j}\left(\log \left(\varphi_{j}(u) \varphi_{j}(v) \varphi_{j}(u)^{*} \varphi_{j}(v)^{*}\right)\right)\right) \\
= & \theta_{j} .
\end{aligned}
$$


By the assumption, $\theta_{j}=\theta, j=1,2$. So the spectrum of $w$ has only one point which is equal to $e^{2 \pi i \theta}$. In other words, $w=e^{2 \pi i \theta}$. It follows that $u v=e^{2 \pi i \theta} v u$. Therefore $A_{u, v}$ is isomorphic to a quotient of $A_{\theta}$.

If $\theta$ is an irrational number, it is well known that irrational rotation algebra $A_{\theta}$ is simple, so $A_{u, v} \cong A_{\theta}$.

Corollary 3.10. Let $A$ be a unital $C^{*}$-algebra and $u, v \in U(A)$ satisfy the condition $\|u v-v u\|<$ 2 and $u v u^{*} v^{*}$ commutes with $u$ and $v$. Let $A_{u, v}$ be the $C^{*}$-subalgebra generated by unitaries $u$ and $v$. If $A_{u, v}$ has a unique tracial state, then $A_{u, v}$ is isomorphic to some irrational rotation algebra $A_{\theta}$, or some matrix algebra $M_{n}$.

Proof. Let $\tau$ be the unique tracial state on $A_{u, v}$. If $\frac{1}{2 \pi i} \tau\left(\log \left(u v u^{*} v^{*}\right)\right)=\theta$ is an irrational number, by Theorem [3.9, $A_{u, v} \cong A_{\theta}$.

If $\frac{1}{2 \pi i} \tau\left(\log \left(u v u^{*} v^{*}\right)\right)=\theta$ is a rational number, then $A_{u, v}$ is isomorphism to a quotient of rational rotation algebra $A_{\theta}$. It follows from [30] that $A_{\theta}$ is strongly Morita equivalent to $C\left(\mathbb{T}^{2}\right)$. Therefore $A_{\theta} \otimes \mathcal{K} \cong C\left(\mathbb{T}^{2}\right) \otimes \mathcal{K}$, where $\mathcal{K}$ is the $C^{*}$-algebra of compact operator on an infinite dimensional separable Hilbert space. Let $\phi: A_{\theta} \otimes \mathcal{K} \rightarrow C\left(\mathbb{T}^{2}\right) \otimes \mathcal{K}$ denote the isomorphism. Then

$$
A_{\theta}=\left(1_{A_{\theta}} \otimes e_{11}\right)\left(A_{\theta} \otimes \mathcal{K}\right)\left(1_{A_{\theta}} \otimes e_{11}\right) \cong \phi\left(1_{A_{\theta}} \otimes e_{11}\right)\left(C\left(\mathbb{T}^{2}\right) \otimes \mathcal{K}\right) \phi\left(1_{A_{\theta}} \otimes e_{11}\right) .
$$

Thus we can find a projection $P_{1} \in M_{N}\left(C\left(\mathbb{T}^{2}\right)\right)$ which is equivalent to $\phi\left(1_{A_{\theta}} \otimes e_{11}\right)$ for some $N \in \mathbb{N}$. So $A_{\theta} \cong P_{1} M_{N}\left(C\left(\mathbb{T}^{2}\right)\right) P_{1}$, where $N$ is an integer and $P_{1} \in M_{N}\left(C\left(\mathbb{T}^{2}\right)\right)$ is a projection. Since each quotient of $P_{1} M_{N}\left(C\left(\mathbb{T}^{2}\right)\right) P_{1}$ is isomorphic to $P_{1} M_{N}(C(X)) P_{1}$ for some closed subset $X \subset \mathbb{T}^{2}$, we have $A_{u, v} \cong P_{1} M_{N}(C(X)) P_{1}$ for some closed subset $X \subset \mathbb{T}^{2}$. The assumption that $A_{u, v}$ has an unique tracial state implies $X$ has only one point. It follows that $A_{u, v} \cong M_{n}$ for some $n \in \mathbb{N}$.

\section{Stability of irrational rotation in infinite simple $C^{*}$-algebras}

Eilers and Loring (Corollary 7.6 of [6]) showed that the answer to (Q1) is affirmative for all rational numbers in $(-1 / 2,1 / 2]$ if the class of unital simple $C^{*}$-algebras of tracial rank zero is replaced by the class of all matrix algebras. As mentioned in the introduction, their result could not include irrational numbers. To include irrational numbers, one may replace $M_{n}$, a finite dimensional simple $C^{*}$-algebra by a unital infinite dimensional simple AF-algebra. To make it even more general, we will replace finite dimensional simple $C^{*}$-algebras (matrix algebras) by unital simple $C^{*}$-algebras with tracial rank zero.

We would also remark an affirmative answer to (Q1) does not follow from Theorem 3.9 even in the additional assumption that $u v u^{*} v^{*}$ commutes with $u$ and $v$. Note that, in Theorem 4.5 and Theorem 5.3, $\tau$ are tracial states on $A$, while $\tau$ in Theorem 3.9 are all tracial states on $A_{u, v}$.

We recall the definition of tracial (topological) rank of $C^{*}$-algebras.

Definition 4.1. ([22]) Let $A$ be a unital simple $C^{*}$-algebra. Then $A$ is said to have tracial (topological) rank zero if for any $\varepsilon>0$, any finite set $\mathcal{F} \subset A$ and any nonzero positive element $c \in A$, there exists a finite dimensional $C^{*}$-subalgebra $B \subset A$ with $1_{B}=p$ such that:

(i) $\|p a-a p\|<\varepsilon$ for all $a \in \mathcal{F}$,

(ii) $\operatorname{dist}($ pap,$B)<\epsilon$ for all $a \in \mathcal{F}$,

(iii) $1_{A}-p$ is Murray-von Neumann equivalent to a projection in $\overline{c A c}$.

If $A$ has tracial rank zero, we write $\operatorname{TR}(A)=0$. 
Definition 4.2. Let $L: A \rightarrow B$ be a linear map. Let $\delta>0$ and $\mathcal{G} \subset A$ be a (finite) subset. We say $L$ is $\mathcal{G}$ - $\delta$-multiplicative if

$$
\|L(a b)-L(a) L(b)\|<\delta \text { for all } a, b \in \mathcal{G} .
$$

We begin with the following lemma which is known.

Lemma 4.3 (Lemma 4.1 of [24]). Let $A$ be a separable unital $C^{*}$-algebra. For any $\epsilon>0$ and any finite subset $\mathcal{F} \subset A_{\text {s.a. }}$, there exists $\delta>0$ and a finite subset $\mathcal{G} \subset A_{\text {s.a }}$ satisfying the following: For any $\mathcal{G}-\delta$-multiplicative contractive completely positive linear map $L: A \rightarrow B$, for any unital $C^{*}$-algebra $B$ with $T(B) \neq \emptyset$, and any tracial state $t \in T(B)$, there exists a $\tau \in T(A)$ such that

$$
|t \circ L(a)-\tau(a)|<\epsilon \text { for all } a \in \mathcal{F} \text {. }
$$

Let $\theta \in(-1 / 2,1 / 2)$ be an irrational number and $\epsilon=\left|1-e^{2 \pi i \theta}\right|$. By recalling 2.6, we write

$$
p_{\epsilon}=\left(a_{i, j}\right)_{K \times K}, \quad q_{\epsilon}=\left(c_{i, j}\right)_{K \times K} \text { and } b_{\epsilon}=\left[p_{\epsilon}\right]-\left[q_{\epsilon}\right]
$$

where $a_{i, j}, b_{i, j} \in \mathcal{T}_{\epsilon}$.

Let $\phi_{\theta}: \mathcal{T}_{\epsilon} \rightarrow A_{\theta}$ be the homomorphism such that $\phi_{\theta}\left(\mathfrak{u}_{\epsilon}\right)=u_{\theta}$ and $\phi_{\theta}\left(\mathfrak{v}_{\epsilon}\right)=v_{\theta}$. Let $A$ be a unital $C^{*}$-algebra and $u, v \in A$ be two unitaries and let $A_{u, v}$ be the $C^{*}$-subalgebra of $A$ generated by $u$ and $v$. If $\|u v-v u\|<\epsilon$, then there is surjective homomorphism $\phi_{u, v}: \mathcal{T}_{\epsilon} \rightarrow A_{u, v}$ such that $\phi_{u, v}\left(\mathfrak{u}_{\epsilon}\right)=u$ and $\phi_{u, v}\left(\mathfrak{v}_{\epsilon}\right)=v$.

Lemma 4.4. Let $\theta \in(-1 / 2,1 / 2)$. For any $\epsilon_{0}>0$, any $\eta_{1}>0$ and any finite subset $\mathcal{G} \subset A_{\theta}$, there exists $\delta_{00}>0$ satisfying the following: For any unital $C^{*}$-algebra $A$ and any pair of unitaries $u, v \in A$, if

$$
\left\|u v-e^{2 \pi i \theta} v u\right\|<\delta_{00},
$$

then there exists a unital $\mathcal{G}-\eta_{1}$-multiplicative contractive completely positive linear map $L: A_{\theta} \rightarrow$ A such that

$$
\begin{aligned}
& \left\|L\left(u_{\theta}\right)-u\right\|<\epsilon_{0}, \quad\left\|L\left(v_{\theta}\right)-v\right\|<\epsilon_{0} \text { and } \\
& \left(\imath \circ \phi_{u, v}\right)_{* 0}\left(\left[p_{\epsilon}\right]\right)=\left[L \circ \phi_{\theta}\right]\left(\left[p_{\epsilon}\right]\right) \\
& \left(\iota \circ \phi_{u, v}\right)_{* 0}\left(\left[q_{\epsilon}\right]\right)=\left[L \circ \phi_{\theta}\right]\left(\left[q_{\epsilon}\right]\right) \text { in } K_{0}(A),
\end{aligned}
$$

where $\imath: A_{u, v} \rightarrow A$ is the unital embedding map. Moreover, if $\theta=p / q \in(-1 / 2,1 / 2]$, where $p$ and $q$ are non-zero integers with $(p, q)=1$ and $q>0$, we may also assume that

$$
[L]\left(\operatorname{bott}\left(u_{\theta}^{q}, v_{\theta}^{q}\right)\right)=\operatorname{bott}\left(u^{q}, v^{q}\right) .
$$

Proof. Let $\eta>0$ be any positive number with $\eta<\epsilon_{0} / 2$ and let $N \geq 1$ be an integer. There is $\delta_{00}>0$ such that if $\left\|u v-e^{2 \pi i \theta} v u\right\|<\delta_{00}$, then there exist a surjective homomorphism $\phi_{u, v}: \mathcal{T}_{\epsilon} \rightarrow A_{u, v}$ such that $\phi_{u, v}\left(\mathfrak{u}_{\epsilon}\right)=u$ and $\phi_{u, v}\left(\mathfrak{v}_{\epsilon}\right)=v$, and a $\mathcal{G}$ - $\eta_{1}$-multiplicative contractive completely positive linear map $L: A_{\theta} \rightarrow A$ such that

$$
\begin{gathered}
\left\|\imath \circ \phi_{u, v}\left(a_{i, j}\right)-L\left(\phi_{\theta}\left(a_{i, j}\right)\right)\right\|<1 /\left(4 K^{2}\right), \\
\left\|\imath \circ \phi_{u, v}\left(c_{i, j}\right)-L\left(\phi_{\theta}\left(c_{i, j}\right)\right)\right\|<1 /\left(4 K^{2}\right)
\end{gathered}
$$

for all $i, j \in\{1,2, \ldots, K\}$.

Moreover, we may also assume that

$$
\left\|L\left(u_{\theta}\right)-u\right\|<\eta / 4 N<\epsilon_{0} \text { and }\left\|L\left(v_{\theta}\right)-v\right\|<\eta / 4 N<\epsilon_{0} .
$$




$$
\left\|L\left(u_{\theta}^{N}\right)-L\left(u_{\theta}\right)^{N}\right\|<\eta / 4 \text { and }\left\|L\left(v_{\theta}^{N}\right)-L\left(v_{\theta}\right)^{N}\right\|<\eta / 4 .
$$

We then obtain that

$$
\begin{aligned}
& \left\|\left[\left(\imath \circ \phi_{u, v}\right) \otimes \operatorname{id}_{M_{K}}\right]\left(p_{\epsilon}\right)-\left[\left(L \circ \phi_{\theta}\right) \otimes \operatorname{id}_{M_{K}}\right]\left(p_{\epsilon}\right)\right\|<1 / 4 \text { and } \\
& \left.\|\left[\left(\imath \circ \phi_{u, v}\right) \otimes \operatorname{id}_{M_{K}}\right]\left(q_{\epsilon}\right)\right)-\left[\left(L \circ \phi_{\theta}\right) \otimes \operatorname{id}_{M_{K}}\right]\left(q_{\epsilon}\right) \|<1 / 4 .
\end{aligned}
$$

It follows that

$$
\left(\iota \circ \phi_{u, v}\right)_{* 0}\left(\left[p_{\epsilon}\right]\right)=\left[L \circ \phi_{\theta}\right]\left(\left[p_{\epsilon}\right]\right) \text { and }\left(\iota \circ \phi_{u, v}\right)_{* 0}\left(\left[q_{\epsilon}\right]\right)=\left[L \circ \phi_{\theta}\right]\left(\left[q_{\epsilon}\right]\right) .
$$

In the case that $\theta=p / q$ as described in the lemma, we choose $N=q$. By (e 4.5), we have

$$
\left\|L\left(u_{\theta}^{q}\right)-u^{q}\right\|<\eta / 2 \text { and }\left\|L\left(v_{\theta}^{q}\right)-v^{q}\right\|<\eta / 2 .
$$

Therefore, with sufficiently small $\eta$, by the definition of the bott element in 2.7, (e 4.4) also holds.

Theorem 4.5. Let $\theta \in(-1 / 2,1 / 2)$ be an irrational number. For any $\epsilon>0$, there exists $\delta>0$ satisfies the following: For any unital simple infinite dimensional $C^{*}$-algebra $A$ with tracial rank zero and any pair of unitaries $u, v \in A$ such that

$$
\left\|u v-e^{2 \pi i \theta} v u\right\|<\delta \text { and } \frac{1}{2 \pi i} \tau\left(\log \left(u v u^{*} v^{*}\right)\right)=\theta
$$

for all $\tau \in T(A)$, there exists a pair of unitaries $\tilde{u}, \tilde{v} \in A$ such that

$$
\tilde{u} \tilde{v}=e^{2 \pi i \theta} \tilde{v} \tilde{u},\|\tilde{u}-u\|<\epsilon \text { and }\|\tilde{v}-v\|<\epsilon .
$$

Proof. Let $\epsilon_{0}=\left|1-e^{2 \pi i \theta}\right|<2$. We will apply Theorem 3.2 of [25]. Let $A_{\theta}$ be the irrational rotation algebra generated by a pair of unitaries $u_{\theta}$ and $v_{\theta}$ such that $u_{\theta} v_{\theta}=e^{2 \pi i \theta} v_{\theta} u_{\theta}$. By [9], $A_{\theta}$ is a unital simple $A \mathbb{T}$-algebra of real rank zero with

$$
\left(K_{0}\left(A_{\theta}\right), K_{0}\left(A_{\theta}\right)_{+},\left[1_{A_{\theta}}\right]\right)=\left(\mathbb{Z}+\mathbb{Z} \theta,(\mathbb{Z}+\mathbb{Z} \theta)_{+}, 1\right) \text { and } K_{1}\left(A_{\theta}\right)=\mathbb{Z} \oplus \mathbb{Z} .
$$

To apply Theorem 3.2 of [25], put $C=A_{\theta}$. Let $\tau$ be the unique tracial state on $C$. For each $t \in T(A)$, define $\gamma: C_{s . a .} \rightarrow \operatorname{Aff}(T(A))$ by $\gamma(c)(t)=\tau(c)$ for all $c \in C_{\text {s.a. }}$ and all $t \in T(A)$, where $C_{\text {s.a. }}$ is the set of all self-adjoint elements of $C$.

Fix $1>\epsilon>0$ and let $\mathcal{F}=\left\{1_{A_{\theta}}, u_{\theta}, v_{\theta}\right\}$. Let $\eta>0, \delta_{0}>0$ (in place of $\delta$ ), $\mathcal{G}_{1} \subset C$ (in place of $\mathcal{G}$ ) be a finite subset, $\mathcal{H} \subset C_{\text {s.a. }}$. be a finite subset and $\mathcal{P} \subset \underline{K}(C)$ be a finite subset required by Theorem 3.2 of [25] for $\epsilon / 2$ (in place of $\epsilon$ ) and $\mathcal{F}$ given.

Note that $\tau \circ \rho_{C}\left(b_{u_{\theta}, v_{\theta}}\right)=\theta$. Therefore $K_{0}(C)$ is generated by $\left[1_{C}\right]$ and $b_{u_{\theta}, v_{\theta}}$. Thus, we may assume, without loss of generality, that $\mathcal{P}=\left\{\left[1_{C}\right], b_{u_{\theta}, v_{\theta}},\left[u_{\theta}\right],\left[v_{\theta}\right]\right\}$.

It follows from Lemma 4.3 that there exists a finite subset $\mathcal{H}_{1} \subset C_{\text {s.a. }}$ and $\delta_{2}>0$ satisfying the following: For any $\mathcal{H}_{1}-\delta_{2}$-multiplicative contractive completely positive linear map $L: C \rightarrow A$, for any unital $C^{*}$-algebra $A$ with $T(A) \neq \emptyset$, and any tracial state $t \in T(A)$, we have

$$
|t \circ L(c)-\tau(c)|<\eta \text { for all } c \in \mathcal{H} .
$$

Let $\mathcal{G}_{2}=\mathcal{H}_{1} \cup \mathcal{G}_{1}$ and let $\delta_{3}=\min \left\{\delta_{1}, \delta_{2}\right\}$. Choose $1>\delta>0$ such that there is a $\mathcal{G}_{2}-\delta_{3}-$ multiplicative contractive completely positive linear map $L: C \rightarrow A$ (for any unital $C^{*}$-algebra A) such that

$$
\left\|L\left(u_{\theta}\right)-u\right\|<\epsilon / 2 \text { and }\left\|L\left(v_{\theta}\right)-v\right\|<\epsilon / 2
$$


for any pair of unitaries $u$ and $v$ in $A$ with $\left\|u v-e^{2 \pi i \theta} v u\right\|<\delta$. Furthermore, by Lemma 4.4, we may also assume, by choosing even smaller $\delta$, that

$$
\begin{gathered}
{\left[L \circ \phi_{\theta}\right]\left(\left[p_{\epsilon_{0}}\right]\right)=\left(\imath \circ \phi_{u, v}\right)_{* 0}\left(\left[p_{\epsilon_{0}}\right]\right) \text { and }} \\
{\left[L \circ \phi_{\theta}\right]\left(\left[q_{\epsilon_{0}}\right]\right)=\left(\imath \circ \phi_{u, v}\right)_{* 0}\left(\left[q_{\epsilon_{0}}\right]\right) .}
\end{gathered}
$$

Now suppose that $A$ is a unital simple $C^{*}$-algebra with tracial rank zero and let $u, v \in A$ be two unitaries such that

$$
\left\|u v-e^{2 \pi i \theta} v u\right\|<\delta \text { and } \frac{1}{2 \pi i} t\left(\log \left(u v u^{*} v^{*}\right)\right)=\theta
$$

for all $t \in T(A)$. Therefore there exists a $\mathcal{G}_{2}-\delta_{3}$-multiplicative contractive completely positive linear map $L: C \rightarrow A$ such that (ex4), (e 4.8) and (e 4.9) hold. Moreover, by the choices of $\delta$ and $\mathcal{G}$,

$$
|t \circ L(c)-\tau(c)|<\eta \text { for all } c \in \mathcal{H} \text { and } t \in T(A) .
$$

It follows from (e4.8) and (e4.9) that

$$
[L]\left(b_{u_{\theta}, v_{\theta}}\right)=\left(\iota \circ \phi_{u, v}\right)_{* 0}\left(b_{\epsilon_{0}}\right) .
$$

Thus, by the Exel trace formula of Theorem 3.5 .

$$
\begin{aligned}
& \rho_{A}\left([L]\left(b_{u_{\theta}, v_{\theta}}\right)\right)(t)=\rho_{A}\left(\left(\imath \circ \phi_{u, v}\right)_{* 0}\left(b_{\epsilon_{0}}\right)\right)(t) \\
= & \rho_{A}\left(\imath_{* 0}\left(b_{u, v}\right)\right)(t)=\frac{1}{2 \pi i} t\left(\log \left(u v u^{*} v^{*}\right)\right)=\theta
\end{aligned}
$$

for all $t \in T(A)$. Define $\kappa: \mathbb{Z}+\mathbb{Z} \theta \rightarrow K_{0}(A)$ by $\kappa([1])=\left[1_{A}\right]$ and $\kappa(\theta)=\imath_{* 0}\left(b_{u, v}\right)$. Since $t\left(\imath_{* 0}\left(b_{u, v}\right)\right)=\theta=\tau\left(b_{u_{\theta}, v_{\theta}}\right)$ for all $t \in T(A), \kappa$ is an order preserving homomorphism. Now by Theorem 5.2 of [25] we have a unital homomorphism $h: A_{\theta} \rightarrow A$ such that

$$
\begin{aligned}
& h_{* 0}=\kappa \\
& h_{* 1}\left(\left[u_{\theta}\right]\right)=[u] \text { and } h_{* 1}\left(\left[v_{\theta}\right]\right)=[v] .
\end{aligned}
$$

It follows from (e e.11) and (e e.12) that

$$
\left.[h]\right|_{\mathcal{P}}=\left.[L]\right|_{\mathcal{P}} .
$$

Moreover, by (e 4.10),

$$
|t \circ L(c)-\gamma(c)(t)|<\eta \text { and }|t \circ h(c)-\gamma(c)(t)|<\eta
$$

for all $c \in \mathcal{H}$ and all $t \in T(A)$. It follows from Theorem 3.2 of [25] that there exists a unitary $W \in A$ such that

$$
\left\|W^{*} h\left(u_{\theta}\right) W-L\left(u_{\theta}\right)\right\|<\epsilon / 2 \text { and }\left\|W^{*} h\left(v_{\theta}\right) W-L\left(v_{\theta}\right)\right\|<\epsilon / 2 .
$$

Let

$$
\tilde{u}=W^{*} h\left(u_{\theta}\right) W \text { and } \tilde{v}=W^{*} h\left(v_{\theta}\right) W .
$$

Then, since $h$ is a homomorphism,

$$
\tilde{u} \tilde{v}=e^{2 \pi i \theta} \tilde{v} \tilde{u} .
$$

By (e4.13) and (e4.7),

$$
\|\tilde{u}-u\|<\epsilon \text { and }\|\tilde{v}-v\|<\epsilon
$$

Remark 4.6. A version of Theorem 4.5 also holds in unital amenable purely infinite simple $C^{*}$-algebras (see [23]). 


\section{$5 \quad$ Stability of rational rotation in infinite simple $C^{*}$-algebras}

Now we consider the case that $\theta$ is a rational number.

Recall that the rational rotation $C^{*}$-algebra associated with the rational number $\theta$ is the universal $C^{*}$-algebra $A_{\theta}$ generated by a pair $u_{\theta}, v_{\theta}$ of unitaries with $u_{\theta} v_{\theta}=e^{2 \pi i \theta} v_{\theta} u_{\theta}$, where $\theta$ is a rational number. When $\theta=0$, then $A_{0} \cong C\left(\mathbb{T}^{2}\right)$. If $\theta \neq 0$, write $\theta= \pm p / q$ with $p, q$ coprime and $0<2 p \leq q$. Let $\lambda=e^{2 \pi i \theta}$, define $q \times q$ matrices

$$
S_{1}=\left(\begin{array}{ccccc}
1 & & & & \\
& \lambda^{1} & & & \\
& & \lambda^{2} & & \\
& & & \ddots & \\
& & & & \lambda^{q-1}
\end{array}\right) \quad \text { and } S_{2}=\left(\begin{array}{ccccc}
0 & & & & 1 \\
1 & 0 & & & \\
& 1 & 0 & & \\
& & \ddots & \ddots & \\
& & & 1 & 0
\end{array}\right)
$$

Then

$$
S_{1} S_{2}=e^{2 \pi i \theta} S_{2} S_{1}
$$

By the universal property, there is a unital homomorphism $\pi^{(0)}: A_{\theta} \rightarrow M_{q}$ such that $\pi^{(0)}\left(u_{\theta}\right)=$ $S_{1}$ and $\pi^{(0)}\left(v_{\theta}\right)=S_{2}$. Since $S_{1}$ and $S_{2}$ generate $M_{q}$, this gives an irreducible representation of $A_{\theta}$. Fix a pair of complex numbers $\left(t_{1}, t_{2}\right) \in \mathbb{T}^{2}$ and choose a pair of $q$-th roots $\left(r_{1}, r_{2}\right) \in \mathbb{T}^{2}$ such that $r_{1}^{q}=t_{1}$ and $r_{2}^{q}=t_{2}$. Define an automorphism $\alpha_{r_{1}, r_{2}}: A_{\theta} \rightarrow A_{\theta}$ such that $\alpha_{r_{1}, r_{2}}\left(u_{\theta}\right)=r_{1} u_{\theta}$ and $\alpha_{r_{1}, r_{2}}\left(v_{\theta}\right)=r_{2} v_{\theta}$. Then $\pi^{(0)} \circ \alpha_{r_{1}, r_{2}}$ also gives an irreducible representation. It is easy to verify that, if $\left(r_{1}^{\prime}, r_{2}^{\prime}\right) \in \mathbb{T}^{2}$ and $\left(r_{1}^{\prime}\right)^{q}=t_{1}$ and $\left(r_{2}^{\prime}\right)^{q}=t_{2}$, then $\pi^{(0)} \circ \alpha_{r_{1}, r_{2}}$ and $\pi^{(0)} \circ \alpha_{r_{1}^{\prime}, r_{2}^{\prime}}$ are unitarily equivalent (by considering permutations of the $q$-th roots). In particular, they have the same kernel $I_{t_{1}, t_{2}}$. Note that

$$
\pi^{(0)} \circ \alpha_{r_{1}, r_{2}}\left(u_{\theta}^{q}\right)=t_{1} \cdot 1_{M_{q}}=\pi^{(0)} \circ \alpha_{r_{1}^{\prime}, r_{2}^{\prime}}\left(u_{\theta}^{q}\right)
$$

and

$$
\pi^{(0)} \circ \alpha_{r_{1}, r_{2}}\left(v_{\theta}^{q}\right)=t_{2} \cdot 1_{M_{q}}=\pi^{(0)} \circ \alpha_{r_{1}^{\prime}, r_{2}^{\prime}}\left(v_{\theta}^{q}\right) .
$$

Therefore, if $\left(t_{1}, t_{2}\right) \neq\left(t_{1}^{\prime}, t_{2}^{\prime}\right)$ in $\mathbb{T}^{2}$, then $I_{t_{1}, t_{2}} \neq I_{t_{1}^{\prime}, t_{2}^{\prime}}$. In particular, they are not unitarily equivalent.

The following lemma will be used in this paper. It is certainly known to many experts and should follow from [30] and discussion in [4] and [2]. To clarify the matter, we include here.

Lemma 5.1. Let $\theta=p / q \in(-1 / 2,1 / 2]$ be a non-zero rational number, where $p$ and $q$ are two integers, $p \neq 0, q>0$ and $(p, q)=1$. Then there exist an integer $N$ and a projection $P \in M_{N}\left(C\left(\mathbb{T}^{2}\right)\right.$ ) (of rank q) and an isomorphism $H: A_{\theta} \rightarrow P M_{N}\left(C\left(\mathbb{T}^{2}\right)\right.$ )P such that $\pi_{\xi} \circ$ $H\left(u_{\theta}^{q}\right)=t_{1} P\left(t_{1}, t_{2}\right)$ and $\pi_{\xi} \circ H\left(v_{\theta}^{q}\right)=t_{2} P\left(t_{1}, t_{2}\right)$ for any $\xi=\left(t_{1}, t_{2}\right) \in \mathbb{T}^{2}$, where $\pi_{\xi}$ is the evaluation map at $\xi$.

Proof. It follows from [30] and the proof of Corollary 3.10 that $A_{\theta} \cong P_{1} M_{N}\left(C\left(\mathbb{T}^{2}\right)\right) P_{1}$, where $N$ is an integer and $P_{1} \in M_{N}\left(C\left(\mathbb{T}^{2}\right)\right)$ is a projection. Let $\psi$ denote the isomorphism.

Let $\pi$ be any irreducible representation of $A_{\theta}$. Let $\lambda=e^{2 \pi i \theta}$. Since $u_{\theta}^{q} v_{\theta}=\lambda^{q} v_{\theta} u_{\theta}^{q}=v_{\theta} u_{\theta}^{q}$. Then $u_{\theta}^{q}$ lies in the center of $A_{\theta}$ and $\pi\left(u_{\theta}\right)^{q}$ lies in the center of $\pi\left(A_{\theta}\right)$, whence $\pi\left(u_{\theta}\right)^{q}$ is a scalar. Similarly, $\pi\left(v_{\theta}\right)^{q}$ is also a scalar. Let $t_{1}, t_{2} \in \mathbb{T}$ such that $\pi\left(u_{\theta}^{q}\right)=t_{1} I$ and $\pi\left(v_{\theta}^{q}\right)=t_{2} I$. Thus $\pi\left(u_{\theta}\right)$ has possible eigenvalues $r_{1} \lambda^{j}$ for $0 \leq j<q$ and for some $r_{1} \in \mathbb{T}$ such that $r_{1}^{q}=t_{1}$. Let 
$E_{j}=E_{\pi\left(u_{\theta}\right)}\left(r_{1} \lambda^{j}\right)$ be the corresponding spectral projections in $\pi\left(A_{\theta}\right)$ (which we do not know that they are non-zero at moment). We may write

$$
\pi\left(u_{\theta}\right)=\sum_{j=0}^{q-1} r_{1} \lambda^{j} E_{j} .
$$

Since

$$
\sum_{k=0}^{q-1}\left(\lambda^{l}\right)^{k}=0
$$

for all $l \in\{1, \ldots, q-1\}$, we obtain that

$$
E_{i}=\frac{1}{q} \sum_{k=0}^{q-1}\left(r_{1} \lambda^{i}\right)^{-k} \pi\left(u_{\theta}\right)^{k} .
$$

Therefore, for $0 \leq i<q$,

$$
\begin{aligned}
\pi\left(v_{\theta}\right) E_{i} & =\frac{1}{q} \sum_{k=0}^{q-1}\left(r_{1} \lambda^{i}\right)^{-k} \pi\left(v_{\theta}\right) \pi\left(u_{\theta}\right)^{k} \\
& =\frac{1}{q} \sum_{k=0}^{q-1}\left(r_{1} \lambda^{i}\right)^{-k} \lambda^{-k} \pi\left(u_{\theta}\right)^{k} \pi\left(v_{\theta}\right) \\
& =\frac{1}{q} \sum_{k=0}^{q-1}\left(r_{1} \lambda^{i+1}\right)^{-k} \pi\left(u_{\theta}\right)^{k} \pi\left(v_{\theta}\right) \\
& =E_{i+1} \pi\left(v_{\theta}\right) .
\end{aligned}
$$

Let $r_{2} \in \mathbb{T}$ such that $r_{2}^{q}=t_{2}$. We then verify that $E_{i j}=\left(\overline{r_{2}} \pi\left(v_{\theta}\right)\right)^{i-j} E_{j}$ are partial isometries for $0 \leq i, j<q$. Since $\left(\overline{r_{2}} \pi\left(v_{\theta}\right)\right)^{q}=I$, it is easy to verify that these form a set of matrix units for $M_{q}$. Moreover,

$$
\pi\left(v_{\theta}\right)=\sum_{j=0}^{q-1} \pi\left(v_{\theta}\right) E_{j}=r_{2} \sum_{j=0}^{q-1} E_{j+1, j},
$$

where we interpret $E_{q, q-1}$ as $E_{0, q}$. Hence $C^{*}\left(\pi\left(u_{\theta}\right), \pi\left(v_{\theta}\right)\right)$ is isomorphic to $M_{q}$. It follows that $E_{i}$ are all one dimensional.

We have just proved that $\pi=\operatorname{Ad} U \circ \pi^{(0)} \circ \alpha_{r_{1}, r_{2}}$ for some unitary $U \in M_{q}$ and with the primitive ideal space $I_{t_{1}, t_{2}}$.

Let $\xi \in \mathbb{T}^{2}$. Define $\pi_{\xi}(a)=a(\xi)$ for all $a \in P_{1} M_{N}\left(C\left(\mathbb{T}^{2}\right)\right) P_{1}$. Then $\pi_{\xi} \circ \psi$ gives an irreducible representation of $A_{\theta}$. From what we have proved, there is $\left(t(\xi)_{1}, t(\xi)_{2}\right) \in \mathbb{T}^{2}$ such that $\pi_{\xi}$ has

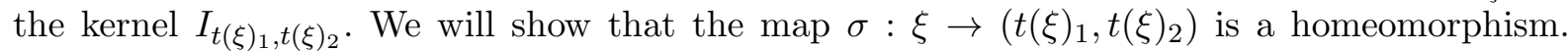
From what described before this lemma, we know that $\sigma$ is injective and, from what we proved above, it is also surjective. Since $\mathbb{T}^{2}$ is a compact Huasdorff space, it suffices to show that $\sigma$ is continuous.

For that, we assume that $\xi_{n} \rightarrow \xi_{0}$ in $\mathbb{T}^{2}$. Then

$$
\begin{aligned}
& \left\|\pi_{\xi_{n}}\left(\psi\left(u_{\theta}^{q}\right)\right)-\pi_{\xi_{0}}\left(\psi\left(u_{\theta}^{q}\right)\right)\right\|=\left\|t\left(\xi_{n}\right)_{1} \cdot 1_{M_{q}}-t\left(\xi_{0}\right)_{1} \cdot 1_{M_{q}}\right\| \rightarrow 0 \text { and } \\
& \left\|\pi_{\xi_{n}}\left(\psi\left(v_{\theta}^{q}\right)\right)-\pi_{\xi_{0}}\left(\psi\left(v_{\theta}^{q}\right)\right)\right\|=\left\|t\left(\xi_{n}\right)_{2} \cdot 1_{M_{q}}-t\left(\xi_{0}\right)_{2} \cdot 1_{M_{q}}\right\| \rightarrow 0 .
\end{aligned}
$$

Therefore

$$
\left|t\left(\xi_{n}\right)_{1}-t\left(\xi_{0}\right)_{1}\right| \rightarrow 0 \text { and }\left|t\left(\xi_{n}\right)_{2}-t\left(\xi_{0}\right)_{2}\right| \rightarrow 0 .
$$


This proves that $\sigma$ is continuous. Therefore $\sigma$ is a homeomorphism. Define $\psi_{1}: P_{1} M_{N}\left(C\left(\mathbb{T}^{2}\right) P_{1} \rightarrow\right.$ $P_{1} M_{N}\left(C\left(\mathbb{T}^{2}\right) P_{1}\right.$ by $\psi_{1}(f)(x)=f\left(\sigma^{-1}(x)\right)$ for all $x \in \mathbb{T}^{2}$ and all $f \in P_{1} M_{N}\left(C\left(\mathbb{T}^{2}\right)\right) P_{1}$, then $\psi_{1}$ is an isomorphism. Put $H=\psi_{1} \circ \psi$. Let $\left(t_{1}, t_{2}\right) \in \mathbb{T}^{2}$ and $y=\sigma^{-1}\left(\left(t_{1}, t_{2}\right)\right)$, i.e., $\left(t(y)_{1}, t(y)_{2}\right)=$ $\left(t_{1}, t_{2}\right)$. Then

$$
\begin{aligned}
\pi_{\left(t_{1}, t_{2}\right)} \circ H\left(u_{\theta}^{q}\right) & =\pi_{\left(t_{1}, t_{2}\right)} \circ \psi_{1} \circ \psi\left(u_{\theta}^{q}\right) \\
& =\pi_{y} \circ \psi\left(u_{\theta}^{q}\right)=t_{1} \cdot 1_{M_{q}}
\end{aligned}
$$

and

$$
\begin{aligned}
\pi_{\left(t_{1}, t_{2}\right)} \circ H\left(v_{\theta}^{q}\right) & =\pi_{\left(t_{1}, t_{2}\right)} \circ \psi_{1} \circ \psi\left(v_{\theta}^{q}\right) \\
& =\pi_{y} \circ \psi\left(v_{\theta}^{q}\right)=t_{2} \cdot 1_{M_{q}} .
\end{aligned}
$$

Put $P=\psi_{1}\left(P_{1}\right)$ and the lemma follows.

Following is a concequence of Theorem 2.7 of [21].

Lemma 5.2. Let $P \in M_{N}\left(C\left(\mathbb{T}^{2}\right)\right.$ ) be a projection of rank $q$ (for some integer $N>q$ ) and let $C=P M_{N}\left(C\left(\mathbb{T}^{2}\right)\right) P$. For any $\epsilon>0$ and finite subset $\mathcal{F} \subset C$, there exists $\delta(\epsilon)>0$ and finite subset $\mathcal{G}(\epsilon)$ satisfies the following: For any unital simple infinite dimensional $C^{*}$-algebra $A$ with real rank zero and stable rank one, if $L: C \rightarrow A$ is a contractive completely positive linear map which is $\mathcal{G}$ - $\delta$-multiplicative and

$$
\left.[L]\right|_{\operatorname{ker} \rho_{\mathrm{C}}} \subset \operatorname{ker} \rho_{A},
$$

then there exists a unital homomorphism $\phi: C \rightarrow A$ such that

$$
\|L(f)-\phi(f)\|<\epsilon \text { for all } f \in \mathcal{F} .
$$

Proof. First consider the case that $C=C\left(\mathbb{T}^{2}\right)$. Note that $K_{0}(C)=\mathbb{Z} \oplus \mathbb{Z}$ with $\operatorname{ker} \rho_{C}=\mathbb{Z}$ (which may be identified with the second copy of $\mathbb{Z}$ ) and $K_{1}(C)=\mathbb{Z} \oplus \mathbb{Z}$. Thus $K L(C, A) \cong$ $\operatorname{Hom}\left(K_{0}(C), K_{0}(A)\right) \oplus \operatorname{Hom}\left(K_{1}(C), K_{1}(A)\right)$. Let $\alpha \in K L(C, A)$. Then, it follows from [19] that there is a unital homomorphism $h: C \rightarrow A$ such that $[h]=\alpha$ if and only if $\alpha\left(\left[1_{C}\right]\right)=\left[1_{A}\right]$ and $\alpha\left(\operatorname{ker} \rho_{C}\right) \subset \operatorname{ker} \rho_{A}$. Thus this theorem follows immediately from Theorem 2.7 of [21] when $C=C\left(\mathbb{T}^{2}\right)$. It is then clear that theorem holds in the case that $C=M_{n}\left(C\left(\mathbb{T}^{2}\right)\right)$ for any integer $n \geq 1$.

For the general case, we note that there exists an integer $N_{1} \geq 1$ and a rank one projection $e \in M_{N_{1}}(C)$ such that $e M_{N_{1}}(C) e \cong C\left(\mathbb{T}^{2}\right)$. Therefore there is a projection $Q \in M_{N_{2}}\left(C\left(\mathbb{T}^{2}\right)\right) \subset$ $M_{N_{1} N_{2}}(C)$ for some integer $N_{2}$, and there is a unitary $W \in M_{N_{2} N_{1}}(C)$ such that $W^{*} Q W=P$. Define $L_{1}=L \otimes \operatorname{id}_{M_{N_{1}}}: M_{N_{1}}(C)=C \otimes M_{N_{1}} \rightarrow A \otimes M_{N_{1}}$. Let $\epsilon_{1}>0$ be given. If $L$ is a $\mathcal{G}$ - $\delta$-multiplicative contractive completely positive linear map with sufficiently small $\delta$ and sufficiently large $\mathcal{G}$, then there exists a unitary $V \in A \otimes M_{N_{1} N_{2}}$ such that

$$
\left\|\left(L \otimes \operatorname{id}_{M_{N_{1} N_{2}}}\right)(W)-V\right\|<\epsilon_{1} .
$$

Then $L_{2}=\left.L_{1}\right|_{e M_{N_{1}}(C) e}$ is close to a unital contractive completely positive linear map $L_{3}$ from $e M_{N_{1}}(C) e \cong C\left(\mathbb{T}^{2}\right)$ into $E M_{N_{1}}(A) E$ for some projection $E \in M_{N_{1}}(A)$ which is close to $L_{2}(e)$, whenever $\delta$ is sufficiently small and $\mathcal{G}$ is sufficiently large. Put $B=M_{N_{2}}\left(e M_{N_{1}}\left(C\left(\mathbb{T}^{2}\right)\right) e\right)$. Then $B \cong M_{N_{2}}\left(C\left(\mathbb{T}^{2}\right)\right)$. Define $L_{4}=L_{3} \otimes \operatorname{id}_{M_{N_{2}}}: B \rightarrow M_{N_{1}}(A) \otimes M_{N_{2}}$. If $L_{4}$ is close a unital homomorphism, say $\psi: B \rightarrow M_{N_{1}}(A) \otimes M_{N_{2}}$, then $\left.L_{4}\right|_{Q B Q}$ is close to $\left.\psi\right|_{Q B Q}$. Note that, there is a unitary $V_{1} \in A \otimes M_{N_{1} N_{2}}$ which is close to $1_{M_{N_{1} N_{2}}}$ such that $V_{1}^{*}\left(V^{*} \psi(Q) V\right) V_{1}=L(Q)=1_{A}$. Therefore $L$ is close to $\left.\operatorname{Ad}\left(V V_{1}\right) \circ \psi\right|_{Q B Q}$. Therefore the general case cne be reduced to the case that $C=M_{n}\left(C\left(\mathbb{T}^{2}\right)\right.$ for some integer $n$. 
Theorem 5.3. Let $\theta \in(-1 / 2,1 / 2)$ be a rational number. Then, for any $\epsilon>0$, there exists $\delta>0$ satisfying the following: For any unital simple $C^{*}$-algebra $A$ with real rank zero and stable rank one and for any pair of unitaries $u$ and $v$ in $A$ such that

$$
\left\|u v-e^{2 \pi i \theta} v u\right\|<\delta \text { and } \frac{1}{2 \pi i} \tau\left(\log \left(u v u^{*} v^{*}\right)\right)=\theta,
$$

for all $\tau \in T(A)$, then there exists a pair of unitaries $\tilde{u}, \tilde{v} \in A$ such that

$$
\tilde{u} \tilde{v}=e^{2 \pi i \theta} \tilde{v} \tilde{u} \text { and }\|u-\tilde{u}\|<\epsilon \text { and }\|v-\tilde{v}\|<\epsilon .
$$

Proof. For the sub-class of simple finite dimensional $C^{*}$-algebras, the theorem follows from Corollary 7.6 of [6]. In what follows we will assume that $A$ is infinite dimensional.

The statement for $\theta=0$ follows from Corollary 2.11 of [21] immediately, or from Lemma 5.2. So, for the rest of the proof, we may assume that $\theta= \pm p / q$, where $p$ and $q$ are non-zero integers with $(p, q)=1,0<2 p<q$. By Lemma 5.1, we may write $A_{\theta}=P M_{N}\left(C\left(\mathbb{T}^{2}\right)\right) P$, where $N$ is an integer and $P \in M_{N}\left(C\left(\mathbb{T}^{2}\right)\right)$ is a projection of rank $q$. Moreover, $\pi_{\xi}\left(u_{\theta}^{q}\right)=t_{1} \circ P_{\xi}$ and $\pi_{\xi}\left(v_{\theta}^{q}\right)=$ $t_{2} P_{\xi}$ for all $\xi=\left(t_{1}, t_{2}\right) \in \mathbb{T}^{2}$. Therefore $\operatorname{ker} \rho_{A_{\theta}}$ is generated by a single element $\operatorname{bott}\left(u_{\theta}^{q}, v_{\theta}^{q}\right)$. Let $\epsilon>0$ and let $\mathcal{F}=\left\{u_{\theta}, v_{\theta}, 1_{A_{\theta}}\right\}$. Let $\delta_{1}>0$ (in place of $\delta(\epsilon)$ ) be a positive number and $\mathcal{G}_{1} \subset A_{\theta}$ (in place of $\mathcal{G}(\epsilon)$ ) be finite subset required by Lemma 5.2 for $C=A_{\theta}=P M_{N}\left(C\left(\mathbb{T}^{2}\right)\right) P, \epsilon / 2$ and $\mathcal{F}$.

Let $\delta_{00}$ be required by Lemma 4.4 for $\epsilon_{0}=\min \left\{\delta_{1}, \epsilon / 2\right\}$ and $\mathcal{G}_{1}$ (in place of $\mathcal{G}$ ). Let

$$
\delta=\min \left\{\delta_{00} / 2 q^{2}, \delta_{0} / 2 q^{2}, 1 / 2 q^{2}\right\},
$$

where $\delta_{0}$ is defined in Proposition 2.8. Suppose that $A$ is a unital simple $C^{*}$-algebra of real rank zero and stable rank one and suppose that $u, v \in A$ are two unitaries such that

$$
\left\|u v-e^{2 \pi i \theta} v u\right\|<\delta \text { and } \frac{1}{2 \pi i} \tau\left(\log \left(u v u^{*} v^{*}\right)\right)=\theta .
$$

It follows from Lemma 4.4 that there exists a unital $\delta_{1}-\mathcal{G}_{1}$-multiplicative contractive completely positive linear map $L: A_{\theta} \rightarrow A$ such that

$$
\left\|L\left(u_{\theta}\right)-u\right\|<\epsilon / 2,\left\|L\left(v_{\theta}\right)-v\right\|<\epsilon / 2 \text { and }[L]\left(\operatorname{bott}\left(u_{\theta}^{q}, v_{\theta}^{q}\right)\right)=\operatorname{bott}\left(u^{q}, v^{q}\right) .
$$

Let $S_{1}, S_{2} \in M_{q}$ be as in (e5.14). Put $U=u \otimes S_{2}$ and $V=v \otimes S_{1}$ in $A \otimes M_{q}$. We compute that

$$
U V=u v \otimes S_{2} S_{1} \approx_{\delta} e^{2 \pi i \theta} v u \otimes\left(e^{-2 \pi i \theta}\right) S_{1} S_{2}=V U
$$

Denote

$$
Z=\left(u \otimes 1_{M_{q}}\right)\left(v \otimes 1_{M_{q}}\right)\left(u^{*} \otimes 1_{M_{q}}\right)\left(v^{*} \otimes 1_{M_{q}}\right)
$$

Then

$$
\begin{aligned}
& \frac{1}{2 \pi i}(\tau \otimes \operatorname{Tr})\left(\log \left(U V U^{*} V^{*}\right)\right) \\
= & \frac{1}{2 \pi i}(\tau \otimes \operatorname{Tr})\left(\log \left(\left(u \otimes S_{2}\right)\left(v \otimes S_{1}\right)\left(u^{*} \otimes S_{2}^{*}\right)\left(v^{*} \otimes S_{1}^{*}\right)\right)\right) \\
= & \frac{1}{2 \pi i}(\tau \otimes \operatorname{Tr})\left(\log \left(Z\left(1_{A} \otimes S_{2}\right)\left(1_{A} \otimes S_{1}\right)\left(1_{A} \otimes S_{2}^{*}\right)\left(1_{A} \otimes S_{1}^{*}\right)\right)\right) \\
= & \frac{1}{2 \pi i}(\tau \otimes \operatorname{Tr})\left(\log \left(Z \cdot e^{-2 \pi i \theta} \cdot 1_{M_{q}(A)}\right)\right)
\end{aligned}
$$


for all $\tau \in T(A)$. Since $e^{-2 \pi i \theta} \cdot 1_{M_{q}(A)}$ is in the center of $M_{q}(A)$, e 5.16 ) equals

$$
\left.\frac{1}{2 \pi i}(\tau \otimes \operatorname{Tr})(\log Z)-q \theta=\frac{1}{2 \pi i}(\tau \otimes \operatorname{Tr})\left(\log \left(u v u^{*} v^{*}\right) \otimes 1_{M_{q}}\right)\right)-q \theta=q \theta-q \theta=0 .
$$

By the Exel trace formula, we conclude that

$$
\operatorname{bott}(U, V) \in \operatorname{ker} \rho_{A} .
$$

It follows that

$$
\operatorname{bott}\left(U^{q}, V^{q}\right)=q^{2} \operatorname{bott}(U, V) \in \operatorname{ker} \rho_{A} .
$$

Note that $U^{q}=u^{q} \otimes 1_{M_{q}}$ and $V^{q}=v^{q} \otimes 1_{M_{q}}$. It follows that

$$
q \operatorname{bott}\left(u^{q}, v^{q}\right)=\operatorname{bott}\left(U^{q}, V^{q}\right) \in \operatorname{ker} \rho_{A} .
$$

This implies that, for all $\tau \in T(A)$,

$$
q \tau\left(\operatorname{bott}\left(u^{q}, v^{q}\right)\right)=0
$$

which implies that

$$
\operatorname{bott}\left(u^{q}, v^{q}\right) \in \operatorname{ker} \rho_{A} .
$$

It follows from (e5.18) and (e5.15) that

$$
[L]\left(\operatorname{bott}\left(u_{\theta}^{q}, v_{\theta}^{q}\right)\right)=\operatorname{bott}\left(u^{q}, v^{q}\right) \in \operatorname{ker} \rho_{A} .
$$

Consequently,

$$
\left.[L]\right|_{\operatorname{ker} \rho_{A_{\theta}}} \subset \operatorname{ker} \rho_{A} .
$$

By applying Lemma 5.2, we obtain a unital homomorphism $\phi: A_{\theta} \rightarrow A$ such that

$$
\left\|L\left(u_{\theta}\right)-\phi\left(u_{\theta}\right)\right\|<\epsilon / 2 \text { and }\left\|L\left(v_{\theta}\right)-\phi\left(v_{\theta}\right)\right\|<\epsilon / 2 .
$$

Put $\tilde{u}=\phi\left(u_{\theta}\right)$ and $\tilde{v}=\phi\left(v_{\theta}\right)$. Note that, since $\phi$ is a unital homomorphism,

$$
\tilde{u} \tilde{v}=e^{2 \pi i \theta} \tilde{v} \tilde{u} .
$$

We also have, by (e 5.19) and (e5.15),

$$
\|\tilde{u}-u\|<\epsilon \text { and }\|\tilde{v}-v\|<\epsilon .
$$

Next we consider that $\theta=\frac{1}{2}$.

Theorem 5.4. For any $1>\epsilon>0$, there exists $\delta>0$ satisfying the following: For any unital simple infinite dimensional $C^{*}$-algebra $A$ with real rank zero and stable rank one and for any pair of unitaries $u$ and $v$ in $A$ such that

$$
\begin{gathered}
\|u v+v u\|<\delta \text { and } \\
\frac{1}{2 \pi i} \tau\left(\log _{0}\left(u v u^{*} v^{*}\right)\right)=1 / 2
\end{gathered}
$$

for all $\tau \in T(A)$, where $\log _{0}$ is a continuous logarithm defined on a compact subset $F$ of $\left\{e^{i t}\right.$ : $t \in(0,2 \pi)\}$ with values in $\{r i: r \in(0,2 \pi)\}$, then there exists a pair of unitaries $\tilde{u}, \tilde{v} \in A$ such that

$$
\begin{gathered}
\tilde{u} \tilde{v}=-\tilde{v} \tilde{u}, \\
\|u-\tilde{u}\|<\epsilon \text { and }\|v-\tilde{v}\|<\epsilon .
\end{gathered}
$$


Proof. The case that $A$ is a unital simple finite dimensional $C^{*}$-algebra follows from Theorem 8.3.4 of [7]. We will consider only infinite dimensional simple $C^{*}$-algebras of real rank zero and stable rank one. The proof is exactly the same as that of Theorem 5.3 except the part to verify (e5.17), i.e.

$$
\operatorname{bott}(U, V) \in \operatorname{ker} \rho_{A} .
$$

In other words, using the Exel trace formula, we need to show that

$$
(\tau \otimes \operatorname{Tr})\left(\log \left(U V U^{*} V^{*}\right)\right)=0 \text { for all } \tau \in T(A) .
$$

We compute that

$$
U V=u v \otimes S_{2} S_{1} \approx_{\delta} e^{2 \pi i \theta} v u \otimes\left(e^{-2 \pi i \theta}\right) S_{1} S_{2}=V U .
$$

We may assume that

$$
\|u v+v u\|<1 / 10
$$

and

$$
\frac{1}{2 \pi i} \tau\left(\log _{0}\left(u v u^{*} v^{*}\right)=1 / 2 .\right.
$$

for all $\tau \in T(A)$. Therefore (by (e5.21) $)$

$$
u v u^{*} v^{*}=\exp (i a)
$$

for some $a \in A_{\text {s.a. }}$ with $\operatorname{spec}(a) \subset(\pi-\pi / 10, \pi+\pi / 10)$. Moreover, by (e 5.22)

$$
\tau(a)=\pi
$$

for all $\tau \in T(A)$.

For any $\tau \in T(A)$, we have

$$
\begin{aligned}
& \frac{1}{2 \pi i}(\tau \otimes \operatorname{Tr})\left(\log \left(U V U^{*} V^{*}\right)\right) \\
= & \frac{1}{2 \pi i}(\tau \otimes \operatorname{Tr})\left(\log \left(\left(u \otimes S_{2}\right)\left(v \otimes S_{1}\right)\left(u^{*} \otimes S_{2}^{*}\right)\left(v^{*} \otimes S_{1}^{*}\right)\right)\right) \\
= & \frac{1}{2 \pi i}(\tau \otimes \operatorname{Tr})\left(\log \left(\left(u v u^{*} v^{*} \otimes 1_{M_{2}}\right) \cdot\left(e^{-\pi i} \cdot 1_{M_{2}(A)}\right)\right)\right) \\
= & \frac{1}{2 \pi i}(\tau \otimes \operatorname{Tr})\left(\log \left(\left(e^{-\pi i / 3} \cdot\left(e^{i a} \otimes 1_{M_{2}}\right)\right) \cdot\left(e^{-\pi i+\pi i / 3} \cdot 1_{M_{2}(A)}\right)\right)\right) .
\end{aligned}
$$

Note that

$$
\begin{aligned}
& \operatorname{spec}\left(\left(u \otimes S_{2}\right)\left(v \otimes S_{1}\right)\left(u^{*} \otimes S_{2}^{*}\right)\left(v^{*} \otimes S_{1}^{*}\right)\right), \quad \operatorname{spec}\left(e^{\frac{-\pi i}{3}} \cdot e^{i a} \otimes 1_{M_{2}}\right), \\
& \text { and } \operatorname{spec}\left(e^{-2 \pi i / 3} \cdot 1_{M_{2}(A)}\right)
\end{aligned}
$$

are all in $\left\{e^{i t}: t \in[-2 \pi / 3, \pi+\pi / 10-\pi / 3]\right\}$.

Since $e^{-\pi i / 3} \cdot e^{i a} \otimes 1_{M_{2}}$ commutes with $e^{-\pi i+\pi i / 3} \cdot 1_{M_{2}(A)}$, we have

$$
\begin{aligned}
& (\tau \otimes \operatorname{Tr})\left(\log \left(\left(e^{-\pi i / 3} \cdot e^{i a} \otimes 1_{M_{2}}\right) \cdot\left(e^{-\pi i+\pi i / 3} \cdot 1_{M_{2}(A)}\right)\right)\right) \\
= & (\tau \otimes \operatorname{Tr})\left(\log \left(e^{-\pi i / 3} \cdot e^{i a} \otimes 1_{M_{2}}\right)\right)+(\tau \otimes \operatorname{Tr})\left(\log \left(e^{-\pi i+\pi i / 3} \cdot 1_{M_{2}(A)}\right)\right) \\
= & (\tau \otimes \operatorname{Tr})\left(\log \left(e^{-\pi i / 3+i a} \otimes 1_{M_{2}}\right)\right)-2 \frac{2 \pi i}{3} \\
= & 2 \tau\left(\frac{-\pi i}{3}+a i\right)-2 \frac{2 \pi i}{3}=0 .
\end{aligned}
$$

It follows that (e-20) holds. 


\section{Acknowledgments}

The first named author was supported by the Zhejiang Provincial Natural Science Foundation of China (Nos. LQ12A01015, LQ13A010016) and by the Research Center for Operator Algebras in East China Normal University. The second named author was partially supported by a grant from East China Normal University and by a NSF grant. The authors acknowledge that they were benefited from conversations with Zhuang Niu. This work was done when both authors were in the Research Center for Operator Algebras in East China Normal University. We would also like to thank Terry Loring who brought our attention to results in [6] and [7].

\section{References}

[1] I. D. Berg and K. R. Davidson, Almost commuting matrices and the Brown-Douglas-Fillmore theorem, Bull. Amer. Math. Soc. (N.S.) 16(1987), no. 1, 97-100.

[2] F. P. Boca, Rotation algebras and continued fractions, Operator algebras, operator theory and applications, 121-142, Oper. Theory Adv. Appl., 181, Birkhäuser Verlag, Basel, 2008.

[3] M. D. Choi, Almost commuting matrices need not be nearly commuting, Proc. Amer. Math. Soc. 102(1988), no. 3, 529-533.

[4] M. D. Choi, G. A. Elliott and N. Yui, Gauss polynomials and the rotation algebra, Invent. math. 99(1990), no. 2, 225-246.

[5] K. R. Davidson, Almost commuting Hermitian matrices, Math. Scand. 56(1985), no. 2, 222240.

[6] S. Eilers and T. A. Loring, Computing contingencies for stable relations, Internat. J. Math. 10(1999), no. 3, 301-326.

[7] S. Eilers, T. A. Loring and G. K. Pedersen, Stability of anticommutation relations: an application of noncommutative CW complexes, J. Reine Angew. Math. 499(1998), 101-143.

[8] S. Eilers, T. A. Loring and G. K. Pedersen, Morphisms of extensions of $C^{*}$-algebras: pushing forward the Busby invariant, Adv. Math. 147(1999), no. 1, 74-109.

[9] G. A. Elliott and D. E. Evans, The structure of the irrational rotation $C^{*}$-algebra, Ann. of Math.(2) 138(1993), no. 3, 477-501.

[10] R. Exel, Rotation numbers for automorphisms of $C^{*}$-algebras, Pacific J. Math. 127(1987), no. $1,31-89$.

[11] R. Exel, The soft torus and applications to almost commuting matrices, Pacific J. Math. 160(1993), no. 2, 207-217.

[12] R. Exel and T. A. Loring, Almost commuting unitary matrices, Proc. Amer. Math. Soc. 106(1989), no. 4, 913-915.

[13] R. Exel and T. A. Loring, Invariants of almost commuting unitaries, J. Funct. Anal. 95(1991), no. 2, 364-376.

[14] P. Friis and M. Rørdam, Almost commuting self-adjoint matrices-a short proof of Huaxin Lin's theorem, J. Reine Angew. Math. 479(1996), 121-131. 
[15] G. Gong and H. Lin, Almost multiplicative morphisms and almost commuting matrices, J. Operator Theory 40(1998), no. 2, 217-275.

[16] P. R. Halmos, Some unsolved problems of unknown depth about operators on Hilbert space, Proc. Roy. Soc. Edinburgh Sect. A 76(1976/77), no. 1, 67-76.

[17] P. de la Harpe and G. Skandalis, Déterminant associé à une trace sur une algébre de Banach. (French) [Determinant associated with a trace on a Banach algebra] Ann. Inst. Fourier (Grenoble) 34(1984), no. 1, 241-260.

[18] R. Høegh-Krohn and T. Skjelbred, Classification of $C^{*}$-algebras admitting ergodic actions of the two-dimensional torus, J. Reine Angew. Math. 328(1981), 1-8.

[19] L. Li, $C^{*}$-algebra homomorphisms and KK-theory, K-Theory 18(1999), no. 2, 161-172.

[20] H. Lin, Almost commuting selfadjoint matrices and applications, Operator Algebras and Their Applications (Waterloo, ON, 1994/1995), Fields Inst. Commun., vol. 13, Amer. Math. Soc., Providence, RI, 1997, 193-233.

[21] H. Lin, When almost multiplicative morphisms are close to homomorphisms, Trans. Amer. Math. Soc. 351(1999), no. 12, 5027-5049.

[22] H. Lin, The tracial topological rank of $C^{*}$-algebra, Proc. London Math. Soc. (3) 83(2001), no. 1, 199-234.

[23] H. Lin, A separable Brown-Douglas-Fillmore theorem and weak stability, Trans. Amer. Math. Soc. 356(2004), no. 7, 2889-2925.

[24] H. Lin, Classification of homomorphisms and dynamical systems, Trans. Amer. Math. Soc. 359(2007), no. 2, 859-895.

[25] H. Lin, The range of approximate unitary equivalence classes of homomorphisms from AHalgebras, Math. Z. 263(2009), no. 4, 903-922.

[26] H. Lin, Asymptotic unitary equivalence and classification of simple amenable $C^{*}$-algebras, Invent. Math. 183(2011), no. 2, 385-450.

[27] T. A. Loring, K-theory and asymptotically commuting matrices, Canad. J. Math. 40(1988), no. $1,197-216$.

[28] T. A. Loring, When matrices commute, Math. Scand. 82(1998), no. 2, 305-319.

[29] M. Pimsner and D. Voiculescu, Exact sequences for K-groups and Ext-groups of certain crossed product $C^{*}$-algebras, J. Operator Theory 4(1980), no. 1, 93-118.

[30] M. A. Rieffel, The cancellation theorem for projective modules over irrational rotation $C^{*}$ algebras, Proc. London Math. Soc. (3) 47(1983), no. 2, 285-302.

[31] P. Rosenthal, Research Problems: Are almost commuting matrices near commuting matrices?, Amer. Math. Monthly 76(1969), no. 8, 925-926.

[32] D. Voiculescu, Asymptotically commuting finite rank unitary operators without commuting approximants, Acta Sci. Math. (Szeged) 45(1983), no. 1-4, 429-431.

hlin@uoregon.edu 